Hier steht eine Anzeige.

Springer

\title{
Schlaganfallprävention
}

\section{Statine in der Primärpävention}

Fragestellung: Können Statine die Häufigkeit kardiovaskulärer Erkrankungen bei Erwachsenen reduzieren?

Hintergrund: Erhöhtes Cholesterin ist ein wichtiger Risikofaktor für die koronare Herzerkrankung und den Herzinfarkt, aber ein deutlich weniger wichtiger Risikofaktor für den Schlaganfall. Unbestritten ist die Wirksamkeit von Statinen in der Sekundärprävention bei Patienten mit akutem Koronarsyndrom (ACS) und nach ischämischem Insult oder transitorischer ischämischer Attacke (TIA). Umstritten ist die Frage, ob Statine auch in der Primärprävention wirksam sind. Dies sollte in der vorliegenden Metaanalyse untersucht werden.

Methodik: Die „US Preventive Services Task Force“ analysierte 19 randomisierte Studien mit insgesamt 71.344 Teilnehmern. Die meisten davon waren im Alter von 51 bis 66 Jahren. Verglichen wurden Statine mit Placebo. Endpunkte waren die Gesamtsterblichkeit, die kardiovaskuläre Sterblichkeit sowie Herzinfarkt und Schlaganfall.

Ergebnisse: Von den analysierten Studien schlossen sechs Patienten mit Hyperlipidämie ein, drei Studien Patienten mit Schlaganfall, vier Studien Patienten mit Diabetes mellitus und zwei Studien Patienten mit arterieller Hypertonie. Am häufigsten wurden Simvastatin und Atorvastatin untersucht. Für die Gesamtsterblichkeit zeigte sich eine signifikante Risikoreduktion um 14\% zugunsten der Statine. Die kardiovaskuläre Sterblichkeit war um 31 \% reduziert, die Schlaganfallhäufigkeit um $29 \%$ und die Herzinfarktrate um 35\%. Für den kombinierten Endpunkt Herzinfarkt, Schlaganfall und kardiovaskulärer Tod betrug die Risikoreduktion $30 \%$. Die Therapieeffekte waren über demografische und klinische Subgruppen hinweg identisch und auch für Patienten mit einem Cholesterinspiegel $<200 \mathrm{mg} /$ dl nachweisbar. Der therapeutische Nutzen war allerdings umso höher, je höher der Cholesterinspiegel war. Unter der Statin-Therapie wurden keine Leberschäden beobachtet. Es ergab sich auch kein erhöhtes Risiko für Diabetes mellitus. Leider untersuchte keine der Studien ein festes Dosis-

Chou R, Dana T, Blazina l et al. Statin use for the prevention of cardiovascular disease in adults: A systematic review for the U.S. Preventive Services Task Force. JAMA 2016; 316: $2008-24$ schema mit einer am Cholesterinspiegel orientierten titrierten Therapie.

Schlussfolgerungen: In einer großen Metaanalyse mit 71.344 Patienten reduzierten Statine signifikant alle wichtigen vaskulären Endpunkte.

\section{- Kommentar von Hans-Christoph Diener, Essen}

\section{Alle vaskulären Endpunkte werden reduziert}

Diese große Metaanalyse zeigt, dass die Patienten über einen weiten Bereich von Cholesterinspiegeln von einer Behandlung mit Statinen profitieren. Dies gilt sowohl für die Primär- als auch für die Sekundärprävention und für alle vaskulären Endpunkte. Herzinfarkte werden etwas besser verhindert als Schlaganfälle. Unstrittig ist die Wirkung der Statine in der Sekundärprävention nach Herzinfarkt und Schlaganfall. Weiterhin ist leider nicht geklärt, ob alle Patienten dieselbe Dosis eines Statins erhalten sollen oder ob ein bestimmter Wert eines LDL-Cholesterins angestrebt werden sollte. Daher werden in den neuen Leitlinien zur Therapie der Hypercholesterinämie im Gegensatz zu früher auch keine Cholesterinplasmaspiegel mehr als Zielwerte angegeben. 